\title{
Status and Outlook for the Dynamic Nuclear Polarization Program at ORNL
}

\section{Josh Pierce*}

Oak Ridge National Laboratory

E-mail: piercejjeornl.gov

The spin dependence of the neutron scattering cross section, especially for hydrogen, makes Dynamic Nuclear Polarization a powerful technique for improving neutron diffraction measurements, especially for biological and soft matter systems. Oak Ridge National Laboratory has demonstrated the application of this technique to Neutron Macromolecular Crystallography, with an eye towards DNP become a normal part of the user program for the Second Target Station that is being built at the SNS. The status of the current system will be discussed.

The 18th International Workshop on Polarized Sources, Targets, and Polarimetry, PSTP2019 23-27 September, 2019

Knoxville, Tennessee

${ }^{*}$ Speaker. 


\section{Introduction}

Macromolecular crystallography has proven to be a powerful tool for exploring the structure of proteins. This technique is primarily utilized at X-ray beamlines, where the high flux generated at modern X-ray sources allows high resolution data to be taken using very small protein crystals. One limitation of X-ray protein crystallography is the relative insensitivity of the X-ray to the hydrogen atom. This insensitivity means that the locations of the hydrogen atoms may not be discernible from X-ray diffraction data even when that data extends to atomic resolution. The solution to the problem is Neutron Macromolecular Crystallography (NMC). The neutron has a large scattering cross section for hydrogen, and is therefore able to determine the locations of the hydrogen atoms within the protein. One limitation of this technique is the comparatively low flux of even the most modern neutron sources. In order to get high resolution data using a neutron beam, the crystals must generally be very large, several orders of magnitude larger than the ones used on X-ray beams. This requirement for large crystals is the major bottle-neck in the application of NMC to a large number of protein systems. Of the number of structures that have been determined with X-rays, only a small fraction have also been determined using neutrons.

The spin dependence of neutron scattering represents a potential avenue to increase the use of NMC, and expand it to more protein systems. Using spin polarized neutrons and polarizing the hydrogen nucleus allow the coherent (signal) and spin-incoherent (noise) cross sections to both be altered, potentially increasing the overall signal to noise ratio of the measurement by several orders of magnitude. Dynamic Nuclear Polarization (DNP) offers mechanism to generate the hydrogen nuclear polarization required to take advantage of this spin dependence.

DNP has been utilized in Small Angle Neuron Scattering (SANS) experiments [1][2], and even some tests of inorganic crystals [3], but protein crystallography has not been attempted. A proof of concept system has been developed [4], and data has been taken on IMAGINE [5], a cold neutron beamline at the High Flux Isotope Reactor (HFIR) at Oak Ridge National Laboratory (ORNL) in 2017.

\section{Spin Dependent Neutron Scattering}

The thermal neutron scattering cross section from identical nuclei in a crystal of $N$ unit cells can be written as in equation 2.1 [6].

$$
\frac{d \sigma}{d \Omega}=N\left(\frac{d \sigma}{d \Omega}\right)_{i n c}+N\left(\frac{d \sigma}{d \Omega}\right)_{c o h} \sum_{i} \exp \left(\mathbf{Q} \cdot \mathbf{r}_{\mathbf{i}}\right)
$$

The coherent (Equation 2.2) cross section represents the part of the scattering containing the diffraction information that allows for the determination of the crystal structure, while the incoherent (Equation 2.3) scattering cross section represents an isotropic scattering that enters as a background to the diffraction measurement. These two cross sections can be written in terms of the neutron polarization $p$, the hydrogen polarization $P$, the spin of the nucleus $I$, the spin-independent part of the scattering amplitude $b_{0}$, and the spin dependent part of the scattering amplitude $b$. The terms $b_{0}$ and $b$ must be determined experimentally for different nuclei. These values are well known for hydrogen and many other nuclei. 


$$
\begin{gathered}
\left(\frac{d \sigma}{d \Omega}\right)_{c o h}=b_{0}^{2}+2 b_{0} b I p P+b^{2} I^{2} P^{2} \\
\left(\frac{d \sigma}{d \Omega}\right)_{i n c}=\frac{b^{2}}{4}\left[I(I+1)-p P I-P^{2} I^{2}\right]
\end{gathered}
$$

Figure 1 shows a comparison of the coherent and incoherent cross sections for hydrogen for the case of a $100 \%$ polarized neutron beam $(p=1)$. At well optimized neutron scattering instruments, the incoherent scattering from hydrogen is the dominant background for hydrogen containing samples. Many experiments use deuterated proteins or solvents in an attempt to reduce this background, but in many cases deuteration may be undesirable or impossible. Generally, full deuteration of a protein is only possible in systems that are expressed in E.Coli. This precludes deuteration of most proteins, although deuterated solvents may still be used. Any experiment would benefit from an increase in the coherent cross section, as it will increase the intensity of the measured diffraction, allowing much smaller samples to be used. The best improvement in signal to noise ratio may be with aligned or anti-aligned polarization, depending on whether the hydrogen incoherent scattering is the dominant background term.

\section{Apparatus}

A prototype system for the purpose of polarizing protein crystals has been constructed and commissioned at ORNL. A full description of the device can be found in Reference [4]. A brief summary is included here.

The small size of the samples (typically less that $1 \mu L$ ) allows the use of lower powered refrigerators and microwave sources. The refrigerator was a commercially purchased LD400 dilution refrigerator ${ }^{1}$, modified by the addition of an in-house built sample space and loading system. The samples were loaded through a gas filled volume, into a Kel-F tube filled with liquid ${ }^{4} \mathrm{He}$. In this version of the device, these spaces were not perfectly isolated, so a large heat load was present from the helium super fluid and gas in the the sample space and the loading volume. This heat load generally gives a lower limit on the sample temperature of about $250 \mathrm{mK}$. The device has since been modified to seal the sample space and reduce the heat-load.

The polarizing magnet used was a cryogen-free, warm bore, vertical $5 T$ solenoid magnet ${ }^{2}$. The system was operated in a "Pseudo Frozen Spin" mode. The relatively high temperature meant that the relaxation times are fairly short, usually on the order of an hour or so. In order to preserve as much polarization as possible, it was critical to go from the polarizing configuration to the data taking configuration as quickly as possible. This was accomplished by lowering the solenoid below the beam height without de-energizing it. The detector could then be moved in and data could be collected in the fringe field of the solenoid. The time between polarizing and data taking mode was on the order of ten minutes (see diagram in Figure 2).

\footnotetext{
${ }^{1}$ BlueFors Cryogenics Oy

${ }^{2}$ Cryomagnetics, Inc.
} 


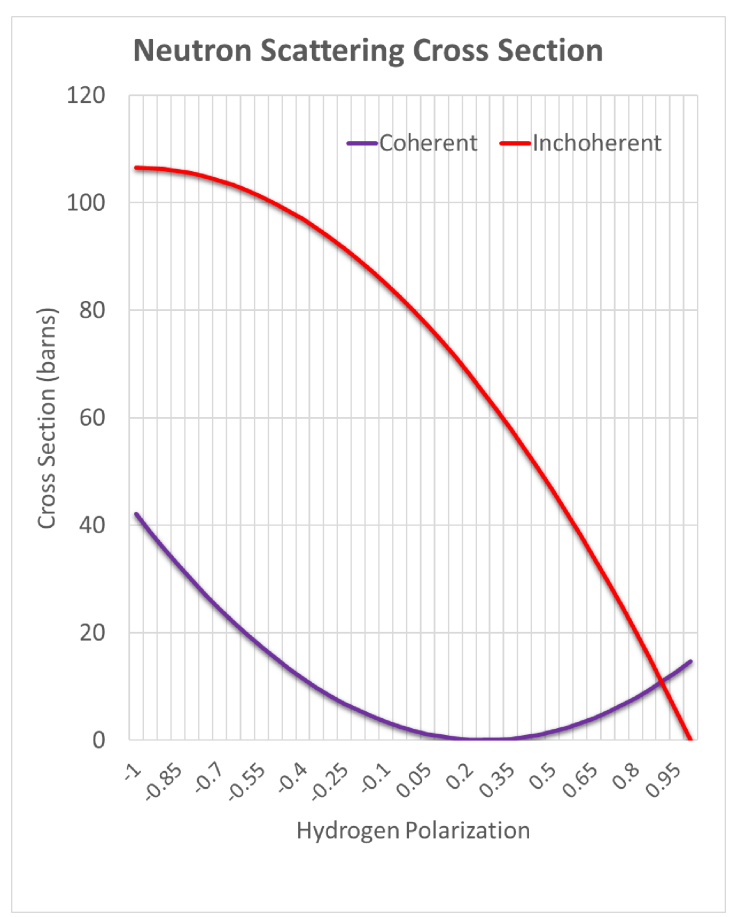

Figure 1: The coherent and incoherent cross sections for a fully polarized neutron scattering off of a polarized hydrogen nucleus, as a function of hydrogen polarization [6]
The microwaves were provided by a solid state $140 \mathrm{GHz}$ microwave source ${ }^{3}$ which was capable of producing up to $300 \mathrm{~mW}$ of microwave power. The NMR system consisted of a Liverpool [8] q-meter tuned to $213 \mathrm{MHz}$. While the q-meter has sufficient sensitivity to monitor the sample polarization, the small sample size meant that the thermal equilibrium signal was too small for a calibration. Larger (noncrystallized) samples containing the solvent and protein in solution were polarized offline in order to estimate the maximum polarization that could be achieved. These tests showed maximum polarization of about $50 \%$.

\section{Detector}

Due to physical incompatibilities with the standard detector system of the IMAGINE instrument, data were collected using a single prototype Anger camera, designed and built by the Detector team in the Neutron Technologies Division at ORNL. This prototype has been designed using SiPM diodes instead of PMTs, allowing it to be used in

the fringe field of the magnet. It was placed at a distance of approximately $20 \mathrm{~cm}$ from the sample position, with a central angle that could be varied between about $15^{\circ}$ and $70^{\circ}$. No background subtraction or "flat field corrections" were done, resulting in images that are visually rather poor, but contained a subset of the diffraction pattern.

\section{Sample Material}

Tests were done using crystals of wild type T4-lysozyme. Crystals must be flash frozen, as slow freezing allows the formation of water crystals which destroy the order in the protein crystal. Smaller crystals are desirable, since the low volume to surface area ratio makes it easy to freeze them by immersion in liquid nitrogen. This is at cross purposes with the normal sample requirements for NMC, where the crystals must be very large in order to compensate for the low neutron flux. In the case of the T4 lysozyme crystals used, studies have been done verifying the diffraction quality of the crystals after the freezing process [7]. Crystals were soaked in a solution

\footnotetext{
${ }^{3}$ Virginia Diode Incorporated
} 
containing $100 \mathrm{mM}$ of hydroxy-TEMPO, with a goal of reaching a final concentration in the crystal similar to the TEMPO concentration used in butanol targets polarized at $5 T$ for nuclear physics experiments[9]. A description of the sample loading scheme can be found in Reference [4].

\section{First Results}
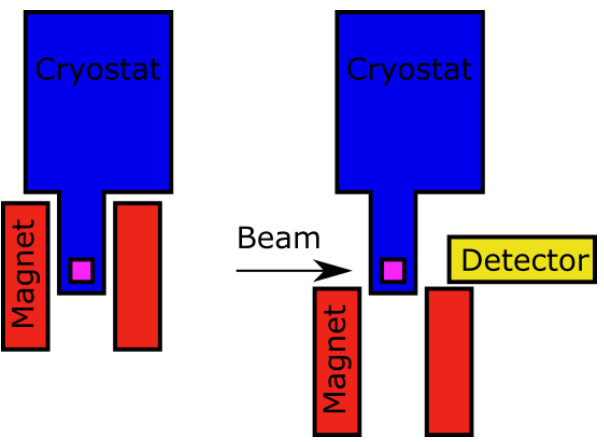

Figure 2: A diagram of the system in data taking mode, showing the magnet lowered to allow the neutron beam to pass over it. After taking data, the magnet would be raised to repolarize the sample. diffraction intensity. These results were consistent across all of the samples, and are also consistent with the assumed 50\% initial polarization. A more thorough discussion of the results of these tests (including diffraction plots) can be found in references [4] and [10]

\section{Outlook}

Another test run was conducted in November of 2019, with modified sample space, and a new detector with better background subtraction. The results of this run are being analyzed, but highlight the potential for DNP to enhance NMC. Efforts are being made to incorporate a DNP system in to the design of the the EWALD diffractometer to be built at the Second Target Station at the Spallation Neutron Source.

\section{Acknowledgments}

Research sponsored by the Laboratory Directed Research and Development Program of Oak Ridge National Laboratory, managed by UT-Battelle, LLC, for the U. S. Department of Energy. This research was done in collaboration with the members of the DyPol LDRD team. The construction and installation of the IMAGINE beam line was partly supported by NSF grant CHE-0922719.

\section{References}

[1] Zhao, J., and H. B. Stuhrmann. "The in situ structure of the L3 and L4 proteins of the large subunit of E. coli ribosomes as determined by nuclear spin contrast variation." Le Journal de Physique IV 3.C8 (1993): C8-233. 
[2] Noda, Y., et al. "Inhomogeneous dynamic nuclear polarization of protons in a lamella-forming diblock copolymer investigated by a small-angle neutron scattering method." Journal of Applied Crystallography 44(3): 503-513. (2011).

[3] Piegsa, F. M., et al. "Polarized neutron Laue diffraction on a crystal containing dynamically polarized proton spins." Journal of Applied Crystallography 46(1): 30-34 (2013).

[4] Pierce, J., et al. "A prototype system for dynamically polarized neutron protein crystallography.", Nuclear Instruments and Methods in Physics Research Section A, 940: 430-434 (2019)

[5] Meilleur, Flora, et al. "The IMAGINE instrument: first neutron protein structure and new capabilities for neutron macromolecular crystallography." Acta Crystallographica Section D: Biological Crystallography 69.10 (2013): 2157-2160.

[6] Abragam, A. Goldman, M. "Nuclear magnetism" Clarendon Press. (1982)

[7] Li, Le, et al. "Neutron crystallographic studies of T4 lysozyme at cryogenic temperature." Protein Science (2017).

[8] G.R. Court, D.W. Gifford, P. Harrison, W.G. Heyes, and M.A. Houlden, Nuclear Instruments and Methods in Physics Research Section A 324 (1993) 433.

[9] C.D. Keith, et al., Nuclear Instruments and Methods in Physics Research Section A 684 (2012) 27.

[10] Pierce, Joshua, et al. "Dynamic nuclear polarization enhanced neutron crystallography: Amplifying hydrogen in biological crystals." Neutron Crystallography in Structural Biology 634 (2020) 153. 